\title{
Enhancing Neurocognitive Assessment Using Immersive Virtual Reality
}

\author{
Thomas D. Parsons \\ Associate Professor \\ Department of Psychology \\ University of North Texas
}

\author{
Susan McPherson \\ Neuropsychologist \\ private practice \\ Edina, Minnesota
}

\author{
Victoria Interrante \\ Associate Professor \\ Department of Computer Science \\ University of Minnesota
}

\begin{abstract}
Immersive virtual reality has significant potential as a tool for enhancing the reliability and specificity of neurocognitive assessment by supporting the ability to carry out carefully controlled, replicable, highly detailed and ecologically valid testing of peoples' ability to perform complex executive functions such as planning and organizing, problem-solving and multitasking, and selective attention and inhibitory control, which are difficult to evaluate using standard paper-and-pencil measures of basic cognitive abilities such as memory, learning, or reasoning. We begin this short paper with an overview of current diagnostic needs in the field of neuropsychology, and a vision of the advantages that immersive virtual reality has the potential to offer as a diagnostic platform. We then briefly summarize our preliminary efforts to develop a robust virtual reality platform for neuropsychological assessment that incorporates a variety of physiological metrics as well as detailed measures of multiple attributes of task performance in a Virtual Environments Grocery Store (VEGS).
\end{abstract}

Keywords: Virtual Reality, Neuropsychology, Rehabilitation, Cognitive Assessment.

Index Terms: I.3.7 [Computer Graphics]: Three-Dimensional Graphics and Realism - Virtual Reality; J.4 [Computer Applications]: Social and Behavioral Sciences - Psychology.

\section{INTRODUCTION}

Accurate neuropsychological testing is critical to the effective management and treatment of cognitive deficits due to brain injuries and illnesses such as Alzheimer's disease. Although a broad battery of tests have been developed to quantitatively assess the nature and severity of various different kinds of cognitive impairments, researchers have long noted that certain types of executive dysfunction that have manifest real-world impact can be notoriously difficult to assess using traditional paper-and-pencil tests. Even real-world observation is not a gold-standard solution in such cases, due to a diversity of concerns including feasibility and safety issues, problems with stimulus control and consistency, and limitations in the scope and detail of the data measures that can be collected. Virtual reality, and immersive virtual reality in particular, has significant promise as a complementary resource for enhancing the accuracy and efficiency of neurocognitive assessment, with broad potential applications. In this short paper, a small team of researchers and clinical practitioners in neuropsychology and virtual reality report some preliminary results from our individual and collaborative efforts to help realize this vision.

\section{BACKGROUND}

Most people receiving rehabilitation for the consequences of brain injury have a number of neuropsychological (cognitive and noncognitive) problems such as deficits in attention, memory, reasoning, and problem-solving. These patients also experience an array of emotional difficulties such as anxiety, depression, and post-traumatic stress disorder. In addition to cognitive and affective issues, behavior problems are notable such as low frustration tolerance, poor self-control, and outbursts of anger. Further subtle motor difficulties are present that may lead to reduced stamina and unsteady gait. In addition, there are often problems connected to social skills and interpersonal relations.

Clinical neuropsychologists working in rehabilitation centers approach the needs of brain-injured people through 1) detailed assessments of various cognitive domains to identify particular deficits as areas to work on in rehabilitation [1]; and 2) targeting of real-life activities of daily living [2]. For the clinical neuropsychologist focused upon rehabilitation, the patient may be asked to perform systematically presented and functionallyoriented therapeutic activities that are based upon an assessment and understanding of the individual's brain-behavior deficits. From a clinical perspective, neurocognitive rehabilitation typically connotes methodical intervention intended to aid the person impacted by cognitive and/or behavioral deficits. In general, the goal is to enable the person to increase his or her ability to perform activities of daily living [3].

Therapeutic interventions by clinical neuropsychologists within rehabilitation settings have traditionally relied upon assessment devices to inform diagnosis and to track changes in clinical status. Although typical assessments employ standard paper-and-pencil psychometrics and training methodologies for impairment assessment and rehabilitation, these approaches have been criticized as limited in the area of ecological validity - the degree of relevance or similarity that a test or training system has relative to the real world and in its value for predicting or improving daily functioning [4-5].

A further common method applied in the rehabilitation sciences employs behavioral observation and ratings of human performance in the real world or via physical mock-ups of functional environments [6]. Activities of daily living within mock-up environments (i.e. kitchens, bathrooms, etc.) and workspaces (i.e. offices, factory settings, etc.) are typically built, within which persons with motor and/or neurocognitive impairments are observed while their performance is evaluated. Aside from the economic costs to physically build these environments and to provide human resources to conduct such evaluations, this approach is limited in the systematic control of real-world stimulus challenges and in its capacity to provide detailed performance data capture. 
The increasing availability of sophisticated technology such as virtual reality (VR) has the potential to enhance our ability to assess various cognitive domains to identify particular deficits and target real-life activities of daily living. The unique match between VR technology assets and the needs of various clinical application areas has been recognized by a number of authors [7, 8] and an encouraging body of research has begun to emerge $[9,10]$. Continuing advances in VR technology along with concomitant system cost reductions have supported the development of more usable, useful and accessible VR systems that can uniquely target a wide range of physical, psychological and cognitive clinical targets and research questions. What makes VR application development in the assessment, therapy and rehabilitation sciences so distinctively important is that it represents more than a simple linear extension of existing computer technology for human use. VR offers the potential to create systematic human testing, training and treatment environments that allow for the precise control of complex, immersive, dynamic three-dimensional (3-D) stimulus presentations, within which sophisticated interaction, behavioral tracking and performance recording is possible. Virtual environments (VEs) can be developed to present simulations that can assess, treat and rehabilitate human functional performance under a range of stimulus conditions that are not easily deliverable and controllable in the 'real-world'.

\section{ASSESSMENT OF EVERYDAY FUnCtioning}

Many neuropsychological tasks have been developed to help localize brain damage, and much progress has been made in elucidating the relationship between specific cognitive function deficits and damage to or deterioration of particular anatomical structures [11]. However, a serious limitation with many traditional neurocognitive tests is that they are not repeatable because of substantial practice effects and cannot be used to assess training outcomes because of low test-retest reliability [12].

In rehabilitation populations, and indeed in many other clinical subgroups as well, there are notable dissociations between neuropsychological test performance and everyday behavior competencies [13]. One problem is that the cognitive tasks found on standard paper-and-pencil tests at times do not appear to fully reflect the range of performance abilities found in the everyday activities [14]. This has led to the proposal that that application of the principles of "ecological validity" is critical to the assessment of cognitive function [15]. The term "ecological validity" refers to the relations between 1) a patient's performance on a set of neuropsychological tests; and 2) the patient's behavior in a variety of real world settings. To establish ecological validity of neuropsychological measures, neuropsychologists focus on demonstrations of either (or both) verisimilitude and veridicality. By verisimilitude, ecological validity researchers are emphasizing the need for the data collection method to be similar to real life tasks in an open environment. For the neuropsychological measure to demonstrate veridicality, the test results should reflect and predict real world phenomena [5].

An example of current approaches to ecological validity by rehabilitation clinicians is found in assessments of behavioral competence in older adults, such as instrumental activities of daily living scales. While these scales may be good predictors of ability to remain independent, they measure impairment rather than a range of ability. They are not specifically designed to assess impairment in activities of daily living relevant to cognitive function, and deficits may be attributable to multiple sources including visual impairments. Hence, they fall short of establishing either verisimilitude or veridicality.
In an early attempt to develop an ecologically valid neuropsychological assessment of everyday functioning, Shallice and Burgess devised a Multiple Errands Test (MET), in which patients are given a set of shopping activities that must be completed in real time in a shopping area [16]. The MET evaluates how patients complete a series of errands requiring organizing and planning. Activities in the MET include following rules about entering particular shops, making purchases to meet a specified budget, and remembering to meet someone at a predetermined time without additional cues. In terms of verisimilitude, the MET is ecologically valid in that the data collection method is performed as the clinician observes (from a distance) the patient performing real life tasks in an open environment. Further, veridicality has been found in the MET through assessment of the predictive ability of its indices to explain a range of "dysexecutive"-related symptoms in everyday life. The main performance indices from the MET have been found to significantly predict severity of everyday life executive problems [17].

Unfortunately, there are a number of limitations found in the MET. First of all, the MET is difficult to use with patients who have the types of mobility and behavioral problems commonly found in patients needing rehabilitation. Further, the MET involves naturalistic observation, in which the examiner observes from a distance and this limits the variety and sophistication of metrics that may be gleaned from the patient's performance.

\subsection{Virtual Environment Grocery Store}

We are currently developing a neuropsychological battery that reflects a range of difficulty and has ecological relevance by using a virtual reality platform [18]. The Virtual Environment Grocery Store (VEGS) offers a platform for neuropsychological assessment and builds upon prior virtual reality applications that focus on component cognitive processes, including attention processes [19], spatial abilities [20], memory [21], and executive functions [22].

The VEGS is as an advanced computer interface that allows the clinician to immerse the patient within a computer-generated simulation that reflects activities of daily living. It involves a number of brief, shopping-type errands that must be completed in a real environment following certain rules that require problem solving. Since the VEGS allows for precise presentation and control of dynamic perceptual stimuli, it has the potential to provide ecologically valid assessments that combine the veridical control and rigor of laboratory measures with a verisimilitude that reflects real life situations. Additionally, the enhanced computation power allows for a range of the accurate recording of neurobehavioral responses in a perceptual environmental that systematically presents complex stimuli. Such simulation technology appears to be distinctively suited for the development of ecologically valid environments, in which three-dimensional objects are presented in a consistent and precise manner. As a result, patients are able to manipulate three dimensional objects in a virtual grocery store that proffers a range of potential task demands.

In the sections that follow, we aim to describe the iterative process that has occurred in the development of an ecologically valid neuropsychological assessment of every day functioning. The specific objectives of this project are to: 1) develop and evaluate the sensitivity and specificity of a virtual measure of prospective memory and multitasking using a real-world task (e.g. remembering to pick up a prescription at the pharmacy); and 2) compare the sensitivity of virtual measures versus traditional neuropsychological paper-and pencil measures of memory and 
cognitive function. In Section 1, we will describe the first iteration of the VEGS which was based on the MET and was developed using NeuroVR. In Section 2, we describe a more advanced platform that builds upon the NeuroVR version of the VEGS to proffer a more usable platform that can also assess prospective memory. The article concludes with a discussion of the future plans for the VEGS in ecologically valid neuropsychological assessment.

\section{NeuroVR Platform of the Vegs}

To assess executive ability, we developed a MET-based VEGS using NeuroVR. The MET-based VEGS, a 3D virtual grocery store environment, was designed to run on a Pentium 4 notebook computer with 1 GB RAM and a 128-MB DirectX 9 compatible graphics card. The MET-based VEGS runs on the open-source NeuroVR VE platform that includes an Editor and a Player that provide an interactive rendering system based on OpenGL. The NeuroVR Editor makes use of Blender and an integrated suite of three dimensional creation tools. Users navigate and interact within the VEGS using the NeuroVR Player (see Figure 1).

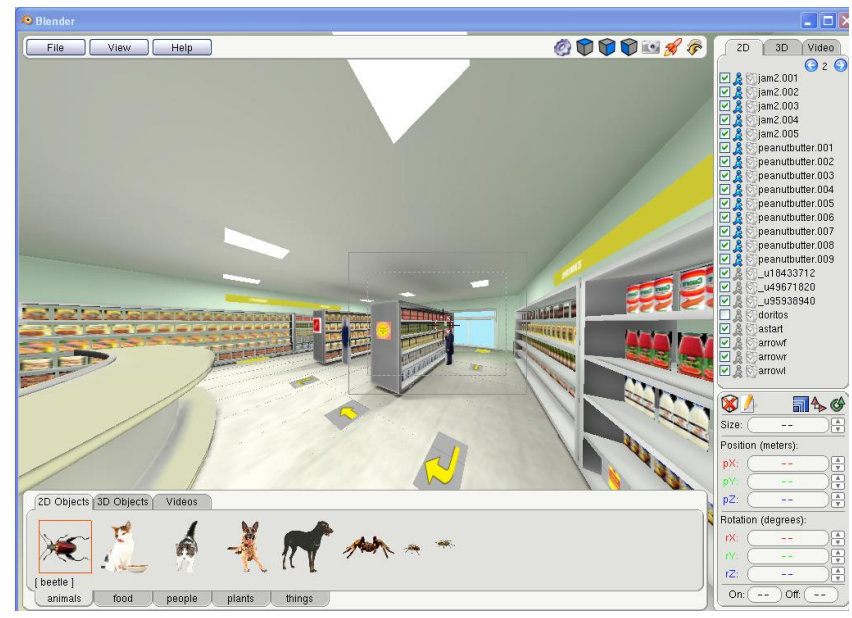

Fig. 1: The NeuroVR Editor. It makes use of Blender and an integrated suite of three dimensional creation tools.

This iteration placed the subject in an immersive modality, in which the VEGS was displayed using a head-tracked eMagin head mounted display. The participant interacted bi-manually within the VEGS using keyboard arrows and a mouse to make selections. Figure 2 shows a participant's view of the VEGS.

The measure we developed involves a number of brief, shopping-type errands that must be completed in a real environment following certain rules that require problem solving. Activities in the VEGS are designed to parallel the MET. There have been other attempts to develop a VR adaptation of the MET. In one adaptation, patients with executive deficits completed fewer tasks than age matched controls, just as they did in the real version of the task [23]. However, no additional conditions were used to increase task demands. In our development of the MET-based VEGS, we aimed to systematically vary the information load (which affects goal maintenance) to offer an advanced VR version of the Multiple Errands Task. We use multiple adaptive trials in the assessment procedure. This is accomplished by creating a pool of "multiple task assignments", empirically determining their baseline difficulty, and then adding conditions

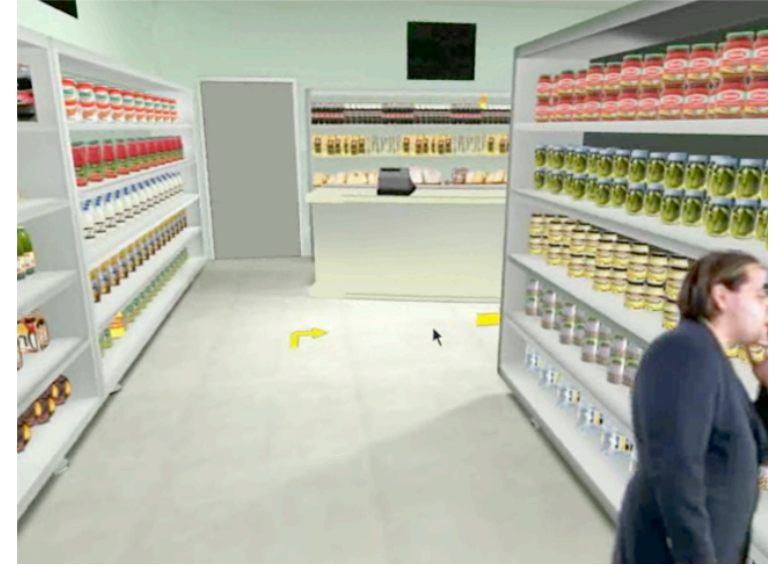

Fig. 2: A view of the VEGS from a participant's perspective.

in the environment that affect baseline task difficulty by manipulating the density of items on shelves, the similarity of packaging, and the intensity and types of realistic irrelevant distractions (e.g. loudness/type of music in the background and loudspeaker announcements). The VEGS platform offers a range of difficulties that may be used to make the tasks sufficiently complex that floor or ceiling effects will not be a problem. Tasks include:

- $\quad$ navigating through a virtual grocery store by following specified routes through the aisles;

- $\quad$ finding and selecting items needed to prepare simple meals, such as making a peanut butter and jelly sandwich;

- pricing and selecting other items so that no more than a budgeted amount is spent;

- $\quad$ performing a prospective memory task when a certain individual is encountered.

Further, the difficulty of tasks assigned is increased over trials by adding distractions:

- increasing the number of items on store shelves

- $\quad$ adding background sounds and music

- $\quad$ increasing the loudness and frequency of distractors

Unfortunately, there were a number of limitations to the NeuroVR platform that needed to be overcome if we were to record a sufficient range of neurobehavioral responses in a perceptual environmental that systematically presents complex stimuli. One of these issues is that we found it difficult to synchronize, in realtime, stimulus data from NeuroVR, behavioral responses from users, and psychophysiological assessments of the users. With NeuroVR, it is difficult to develop a transfer system that would allow access to the data being acquired into a graph by the psychophysiological assessment system. Further, it was difficult to allow third party applications to tap into the data streams being generated by the psychophysiological hardware, Matlab, and NeuroVR during data acquisition.

Knowledge of the user-state during exposure to the VEGS is imperative for the development and assessment of virtual environment design. Different individuals will invariably have different reactions to the VEGS, and without an assessment tool that can be employed online, the clinician will experience difficulties in identifying the causes of these differences, which may lead to a loss of experimental control of the clinical research paradigms. For example, a user may become increasingly frustrated with some aspect of the VEGS, but without proper measurement techniques to detect this frustration while it occurs, compensatory measures cannot be taken and the user's sense of presence, or feeling of "being there," may be diminished. While 
the NeuroVR version of the VEGS offers the capability of presenting a realistic simulation of the real world, online assessment of the user's reactions to that environment is vital to maintaining an understanding of how the environment is affecting the user to preserve experimental control.

One answer to these issues and limitations is the addition of psychophysiological metrics to the VEGS platform. The psychophysiological signal is continuously available, whereas behavioral data alone may be detached from the user's experience and assessed intermittently. The continuous nature of psychophysiological signals is important for several reasons. First, it allows for greater understanding of how any stimulus in the environment impacted the user, not only those stimuli that were targeted to produce behavioral responses [24]. The addition of psychophysiological metrics to the VEGS platform is important because it allows for a continuous objective measure of the user's state, which can include measures of cognitive workload $[25,26]$, varying stress levels [27, 28], task engagement [29, 30], and arousal [31-33] among others. Additionally, multiple channels of psychophysiological data can be gleaned from various sensors simultaneously, which further increases experimental control by providing a combination of measures, so that one measure alone is not the sole basis for design decisions [34].

\section{Second Generation Vegs Platform}

To overcome these interoperability issues, and increase the flexibility of user interaction in the VEGS, we decided to port the VEGS to a new platform that we custom-developed using the G3D [35] graphics engine. We imported a variant of the virtual grocery store model from NeuroVR as a set of textured objects in .obj format and re-designed the locomotion and interaction metaphors to be more compatible with an immersive virtual reality experience.

We used an nVisorSX head mounted display (HMD) to present participants with an immersive view of the virtual environment in stereo over a $60^{\circ}$ diagonal field of view on twin SXGA OLED displays with $100 \%$ stereo overlap. The virtual environment was rendered in real time on a custom-built PC with an nVidia Quadro FX 5800 graphics card. We attached $19 \mathrm{~mm}$ and $14 \mathrm{~mm}$ retroreflective tracking markers, respectively, to the HMD and to a fingerless bicycle glove that participants wore on their dominant hand, allowing the head and hand to each be tracked in real time using a collection of 12 Vicon MX40+ cameras focused on a small region in the center of our lab. The hand-tracking was used to enable participants to interact with objects in the virtual environment by reaching out and touching them. We used a high fidelity rigid hand-and-arm model to represent the location of the hand to participants in as realistic a way as our tracking implementation allowed.

Participants controlled their viewpoint in the virtual environment using a Logitech Attack 3 joystick that was firmly mounted to a short wooden bar that extended forward beneath the armrest of an Aeron chair. We prepared left- and right-handed versions of the chairs, as well as of the gloves and hand models, so that each participant could both drive and select objects with their dominant hand. Participants were able to use the joystick to translate their viewpoint forward and backwards in the virtual environment, but they had to swivel in the chair to turn. We adopted this locomotion model in light of research showing that people are better able to maintain their sense of direction while traveling around in an immersive virtual environment when they are able to physically turn their own bodies within the VE rather than having to virtually turn the VE around themselves while remaining in a fixed physical orientation [36]. While participants were in the VEGS, an audio background consisting of ambient grocery store-like sounds was presented via built-in headphones attached to the HMD. We used physiological sensors from Thought Technology along with Biograph Infiniti software to stream time-stamped EKG and galvanic skin response data to a file. We were able to synchronize this physiological data output stream with an event-logging output stream from our virtual environments software by using a Windows event hook in the Biograph software to time-stamp a key-press event on the single computer that was running both systems.

In order to acclimate participants to the experience of using the virtual environments equipment, we began by using the HMD to immerse them in a high fidelity virtual replica of our lab space. Our goal in doing this was to foster the illusion that VR technology works in a way that is analogous to a magical seethrough camera. Previous research has shown that people are able to achieve a higher sense of presence and a more accurate spatial understanding of a remote virtual environment when they are smoothly transitioned into that environment after first being immersed in a virtual replica room [37]. Participants were required to successfully perform a simple set of flexibly specified navigation and selection tasks in this transitional environment before continuing on to the next phase of the experiment to ensure that they understood how to use the joystick to move around, and their hand to pick things. A second short set of more specifically constrained tasks was then presented, still in the transitional environment, to verify that participants were able to successfully understand and follow instructions.

Finally, participants were transitioned from the virtual replica room into the virtual grocery store environment. Our preliminary implementation in the new VEGS focused specifically (and narrowly) on assessing prospective memory in the elderly. We instantiated two prospective memory tasks: a time-based task, in which participants had to remember to drop off a prescription at the pharmacy when it opened at 9:00am after beginning to shop for groceries at 8:55am; and an event-based task, in which participants had to remember to pick up their prescription from the pharmacy when their number was called over the loudspeaker, while finishing their shopping task. The pharmacy was embedded as a separate counter within the grocery store, shown in figure 3.

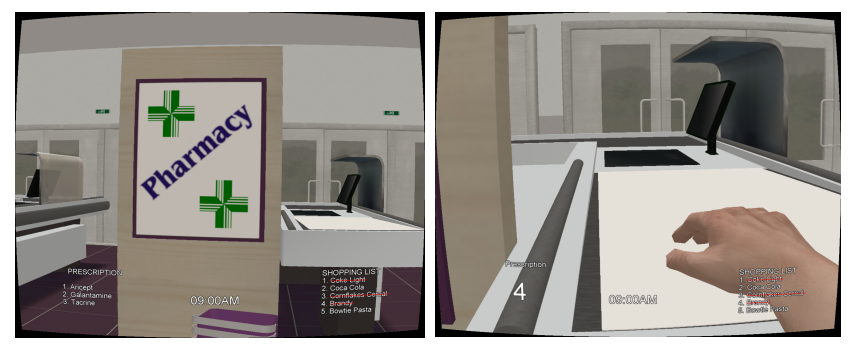

Fig. 3: A view of the pharmacy within our second generation VEGS. Participants had to remember to drop off a prescription at a specified time and then come back to pick it up when their number was called.

Interaction with the virtual environment was done using hand gestures. As a distractor task, participants were asked to shop for a small set of items specified on a provided list. When they found one of the items on a shelf in the supermarket, they could choose it by reaching out to touch it with their hand. When the hand model intersected the object model, the object disappeared from the shelf and a red line was drawn through that item on the shopping list. Figure 4 shows what this looked like. We prepared three levels of lists, containing items that were progressively more difficult to find, to ensure that no participant would run out of items to search for while at the same time allowing some easy initial successes to keep people engaged and avoid frustration. 

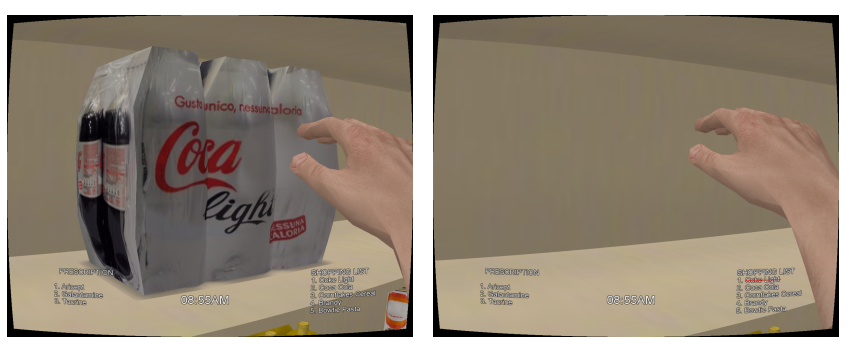

Fig. 4: Object selection, by a right-handed person, in our second generation VEGS.

Although this represents a new platform, the new iteration of the VEGS maintained the neuropsychological characteristics of the original MET-based VEGS. The neuropsychological assessments included both a time-based and an event-based prospective memory task. A written list of shopping items was provided in order to counter the impact of impaired memory in the distractor task. The scenario was designed to accurately resemble real-world procedure. For the time-based measure, two points were awarded for turning in the prescription within two minutes of the time limit, and one point for turning the prescription in after a oral reminder. For the event-based measures three points were awarded for returning to the pharmacy within two minutes of hearing their number called the first time, two points for retrieving the prescription within two minutes of the second announcement, and one point following an on-screen reminder.

\section{Planned Validation Protocol}

Proper scientific analysis of the psychometric properties of the VEGS and the ultimate value of its use in the rehabilitation setting will require detailed study on large numbers of participants with and without symptoms of cognitive decline. A clinical examination of the VEGS involves a systematic evaluation designed to elicit pathologic signs or symptoms. While the VEGS aims to move beyond standard paper-and-pencil tests, it is important that the VEGS have at least the predictive validity found in these tests. Paper-and-pencil neuropsychological tests offer the advantage of being standardized in their administration, materials, and scoring. The development and standardization of VEGS will require a number of steps: 1) establishing the cognitive construct or symptom to be evaluated (e.g., executive functioning; prospective memory); 2) developing the procedures to be administered; and 3) performing a series of validation studies to determine the quality of the information the VEGS provides.

In the rehabilitation center, patients often have difficulties with processing and retaining information. Historically, neuropsychologists investigated the underlying features of these cognitive disturbances using a number of paper-and-pencil tests of sustained attention, learning, and recall to be used as objective measures of these often vaguely defined symptoms. For the past 60 to 100 years validation studies have been performed on these measures to determine whether the tests are able to properly assess the cognitive domain in question and whether they are able to accurately identify impairments in patients with verified brain dysfunction.

For the VEGS, it will be important to establish its psychometric reliability and validity. Psychometrics is the study of psychological tests and measurements, to evaluate the scientific quality of the neuropsychological measures. Reliability refers to the consistency or stability of test scores when they are obtained for an individual after repeated observations or under different testing conditions. Validity refers to how well an instrument measures what it is supposed to be measuring. The performance of a validation study on a neuropsychological test like the VEGS requires sampling an adequate number of subjects to demonstrate the efficacy of the measure.

\subsection{Experimental Protocol}

Experimental sessions will take place over a two hour period. After informed consent is obtained, basic demographic information and computer experience and usage activities are recorded. Strict exclusion criteria will be enforced to minimize possible confounding effects of comorbid factors known to adversely impact cognition, including psychiatric conditions (e.g., mental retardation, psychotic disorders, diagnosed learning disabilities, attention deficit hyperactivity disorder, and bipolar disorders, as well as substance-related disorders within 2 years of evaluation) and neurologic conditions (e.g., seizure disorders, closed head injuries with loss of consciousness greater than 15 minutes, and neoplastic diseases).

Participants complete a thorough neuropsychological assessment (both the VEGS and traditional paper-and-pencil tests) administered under standard conditions, then complete a simulator sickness questionnaire, which includes a pre-VR exposure symptom checklist. Both patients and controls receive the following measures: (1) a 90 minute neuropsychological screening battery (see below), and (2) the VEGS assessment of prospective memory. Test administrations may be counterbalanced with participants randomized so that half receive the neuropsychological battery followed by the VEGS testing and half complete the VEGS testing first. A minimum 30 minute rest period will separate the neuropsychological battery and VR testing.

All participants will be administered the VEGS as part of a larger neuropsychological test battery. Electrocardiographic (ECG), and electrodermal activity (EDA) will be recorded simultaneously throughout the experiment using psychophysiological equipment and a computer running MATLAB software.

\subsection{Neuropsychological Validation}

The following traditional neuropsychological measures will be used: 1) Hopkins Verbal Learning Test-Revised (HVLT-R); 2) Brief Visuospatial Memory Test-Revised (BVMT-R); 3) Controlled Oral Word Association Test (FAS); 4) Semantic Fluency (Animals); 5) Digit Symbol Coding; 6) Digit Span (Forward and Backward) from the Wechsler Adult Intelligence Scale, Third Edition (WAIS III); 7) Trail Making Test Parts A and B (TMT); 8) Stroop Color and Word Test; 9) Wechsler Test of Adult Reading (WTAR); and two measures of prospective memory (the Belonging and Appointment subtests of the Rivermead Behavioral Memory Test-II).

A primary aim of the validation study will be to examine the construct validity of the VEGS using the methodology provided by the multitrait-multimethod matrix [38]. The use of this matrix approach with multiple neurocognitive measures allows the simultaneous investigation of convergent validity (i.e., extent to which different neurocognitive measures of prospective are related) and discriminant validity (i.e., extent to which neurocognitive measures of domains other than prospective memory are unrelated). The use of the multitrait-multimethod matrix gives us the advantage of being able to examine method variance (i.e., degree to which scales are correlated because they use the same method of measurement rather than because they share valid trait variance). Using "pure" measures of memory 
introduces less noise into the decision matrix [39]. Following the multitrait-multimethod matrix, any measure purporting to quantify a particular neurocognitive domain should be highly correlated with other measures of the same neurocognitive domain (convergent validity), whereas it should not be too highly correlated with tests of different neurocognitive domains (discriminant validity).

\subsection{Psychophysiological Validation}

Psychophysiological metrics proffer the advantage of an objective measure of response that can be recorded in realtime as the environment is experienced, providing a continuous measure of presence. Clinical researchers may study the impact of being immersed in the VEGS by looking at the psychophysiological responses of participants to their surroundings [40]. The recording of psychophysiological variables while participants operate within VEs has produced useful results in studies examining presence and immersion [41, 42]. As such, the VE assets that allow for precise stimulus delivery within ecologically enhanced scenarios appears well matched for this research.

Psychophysiological metrics will be used to establish knowledge of the user-state during exposure to the VEGS. EDA will be measured with the use of $8 \mathrm{~mm}$ silver-silver chloride electrodes placed on the volar surface of the distal phalanges of the index and middle fingers of the non-dominant hand. Electrodes will be filled with a 0.05 molar isotonic $\mathrm{NaCl}$ paste to provide a continuous connection between the electrodes and the skin. Skin conductance responses will be scored as the largest amplitude response beginning in a window of 1 to $3 \mathrm{~s}$ following stimulus onset. A response may be defined as having amplitude greater than $0.01 \mu \mathrm{S}$.

ECG will be recorded with use of a Lead 1 electrode placement. Electrode sites are cleaned with alcohol prep pads in order to improve contact. Inter-beat Intervals (IBIs) will be scored as the time difference between successive R waves in the ECG signal. IBIs will $b$ used as the dependent variable analyzed instead of heart rate because of a lowered susceptibility to artifact due to differences in baseline values. A window of $3 \mathrm{~s}$ pre-stimulus onset to $5 \mathrm{~s}$ beginning at stimulus onset will be scored. Instantaneous IBIs will be recorded at half second intervals during the pre- and post-stimulus time windows. A difference score between the average pre-stimulus IBI for each trial and each post-stimulus IBI value will be computed for each trial.

\section{Discussion}

The aim of the current program of research is to develop a virtual reality based grocery store that can be used for detailed assessments of various cognitive domains to identify particular deficits as areas to work on in rehabilitation; and targeting of reallife activities of daily living. Before the VEGS can be fully implemented by clinical neuropsychologists in rehabilitation settings, it must be fully validated against the "gold standard" of neuropsychological assessments found in traditional paper-andpencil assessments. Our goal is to conduct an initial pilot study to validate the VEGS through the use of a standard paper and pencil neuropsychological battery for the assessment of healthy participants. Further, we aim to assess arousal and engagement using various psychophysiological measures. We believe that this will provide a first step in the development of this tool. Many more steps are necessary to continue the process of test development and to fully establish the VEGS as a measure that contributes to existing assessment procedures for the diagnosis of neurocognitive decline.
The VEGS offers a platform for neuropsychological assessments and builds upon prior developments of virtual reality applications that focus on component cognitive processes. The increased ecological validity of neurocognitive batteries that include assessment using virtual scenarios like the VEGS may aid differential diagnosis and treatment planning. Within the VEGS, it is possible to systematically present cognitive tasks targeting neuropsychological performance beyond what are currently available using traditional methods. Reliability of the planned rehabilitation regimens based on neuropsychological assessment can be enhanced in the VEGS by better control of the perceptual environment, more consistent stimulus presentation, and more precise and accurate scoring. The VEGS may also improve the validity of neurocognitive measurements via the increased quantification of discrete behavioral responses, allowing for the identification of more specific cognitive domains. The VEGS could allow for neurocognition to be tested in situations that are more ecologically valid. Participants can be evaluated in an environment that simulates the real world, not a contrived testing environment.

Simulation of real world environments increases the ecological validity of the assessment task while maintaining control of manipulations that can affect performance. Even though participants are quite aware that the VEGS is a virtual reality they willingly "play along" as though the environments are real [18]. Further, it has been argued that reality is experiential, not based on the external environment, and that immersive virtual reality tasks may produce subjective engagement that is equivalent to engagement in the real world. For the clinical neuropsychologist focused upon rehabilitation, the VEGS offers the opportunity to immerse the patient in an ecologically valid environment and observe the patient as she or he performs systematically presented and functionally-oriented therapeutic activities that are based upon an assessment and understanding of the individual's brainbehavior deficits. From a clinical perspective, neurocognitive rehabilitation using a virtual environment like the VEGS allows for methodical assessment and intervention in activities of daily living that will aid the person impacted by cognitive and/or behavioral deficits. As a result, the clinician and patient can work together in a controlled environment that mimics real-world functioning to enable the patient to increase his or her ability to perform activities of daily living.

\section{ACKNOWLEDGMENTS}

Our research involving the second generation VEGS was supported by a grant-in-aid from the Office of the Vice President of Research at the University of Minnesota, and by the $\mathrm{U}$ of $\mathrm{M}$ Foundation through the Linda and Ted Johnson Digital Design Consortium Endowment and Lab Setup Funds. The second generation VEGS was implemented in its initial form by Prince Bajracharya, with some assistance from Amelia Nybakke and Ramya Ramakrishnan. A later version was re-implemented by Peng Liu using the OGRE gaming platform for better real time performance.

\section{REFERENCES}

[1] Caramazza A., Hillis A.E. (1993). For a theory of remediation of cognitive deficits. Neuropsychological Rehabilitation, 3(3), 217-234.

[2] Razani, J., Casas, R., Wong, J. T., Lu, P., Alessi, C., Josephson, K. (2007). Relationship between executive functioning and activities of daily living in patients with relatively mild dementia. Applied Neuropsychology, 14, 208-214. 
[3] Wilson B.A., Herbert C.M., Shiel A., eds. (2003). Behavioural Approaches in Neuropsychological Rehabilitation: Optimising Rehabilitation Procedures. Hove, UK: Psychology Press.

[4] Wilson B.A. (1998). Cognitive rehabilitation: How it is and how it should be. Journal of the International Neuropsychological Society, 3 , 487-496.

[5] Parsons T.D. (2011). Neuropsychological assessment using virtual environments: enhanced assessment technology for improved ecological validity. In S. Brahnam (Ed.), Advanced Computational Intelligence Paradigms in Healthcare: Virtual Reality in Psychotherapy, Rehabilitation, and Assessment (pp. 271- 289). Germany: Springer-Verlag.

[6] Weiss P., Jessel A.S. (1998). Virtual reality applications to work. Work, 11, 277-293.

[7] Rose F.D., Brooks B.M., Rizzo A.A. (2005). Virtual reality in brain damage rehabilitation: review. Cyberpsychology and Behavior, 8 , 241-262.

[8] Parsons T.D., Rizzo A.A., Rogers S.A., York P. (2009). Virtual reality in pediatric rehabilitation: a review. Developmental Neurorehabilitation, 12, 224-238.

[9] Lange B.S., Koenig S., Chang C., McConnell E., Suma E., Bolas M., Rizzo A. (2012). Designing informed game-based rehabilitation tasks leveraging advances in virtual reality. Disability and Rehabilitation, 34, 1863-1870.

[10] Rizzo A.A., Schultheis M.T., Kerns K., Mateer C. (2004). Analysis of assets for virtual reality applications in neuropsychology. Neuropsychology of Rehabilitation, 14, 207-239.

[11] MacPherson S.E., Phillips L.H., Della Sala S. (2002). Age, executive function, and social decision making: a dorsolateral prefrontal theory of cognitive aging. Psychology and Aging, 17(4), 598-609.

[12] O'Neil-Pirozzi T.M., Goldstein R. (2005). Test-retest reliability of the Virtual Planning Test. Brain Injury, 19, 1191-6.

[13] Garden S.E., Phillips L.H., MacPherson S.E. (2001). Midlife aging, open-ended planning, and laboratory measures of executive function. Neuropsychology, 15, 472-482.

[14] Moore D.J., Palmer B.W., Patterson T.L., Jeste D.V. (2007). A review of performance-based measures of functional living skills. Journal of Psychiatric Research, 41(1), 97-118.

[15] Burgess P.W., Alderman N., Forbes C., Costello A., Coates L.M-A., et al., (2006). The case for the development and use of "ecologically valid" measures of executive function in experimental and clinical neuropsychology. Journal of the International Neuropsychological Society, 12(2), 194-209.

[16] Shallice T., Burgess P.W. (1991). Deficits in strategy application following frontal lobe damage in man. Brain, 114, 727-741.

[17] Urbanoa G.C., Caracuelbc A., Lópezac R.C., Serranoa C.V., Batemande A., García A.V. (2013). Ecological validity of the Multiple Errands Test using predictive models of dysexecutive problems in everyday life. Journal of Clinical and Experimental Neuropsychology, 35, 329-336.

[18] Parsons T.D., Rizzo A.A., Brennan J., Zelinski E.A. (2008). Assessment of executive functioning using virtual reality: Virtual Environment Grocery Store. Gerontechnology, 7, 187-191.

[19] Parsons T.D., Bowerly T., Buckwalter J.G., Rizzo A.A. (2007). A controlled clinical comparison of attention performance in children with ADHD in a virtual reality classroom compared to standard neuropsychological methods. Child Neuropsychology, 13, 363-381.

[20] Parsons T.D., Larson P., Buckwalter J.G., Rizzo A.A. (2004). Sex differences in mental rotation and virtual reality spatial rotation. Neuropsychologia , 42, 4, 555-562.

[21] Parsons T.D., and Rizzo A.A. (2008). Initial validation of a virtual environment for assessment of memory functioning: virtual reality cognitive performance assessment test. Cyberpsychology and Behavior, 11, 17-25.
[22] Parsons T.D., Courtney C., Arizmendi B., Dawson M. (2011). Virtual reality Stroop task for neurocognitive assessment. Studies in Health Technology and Informatics, 143, 433-439.

[23] McGeorge P., Phillips L.H., Crawford J.R., Garden S.E., Della Sala S., Milne A.B., et al. (2001). Using virtual environments in the assessment of executive dysfunction. Presence, 10(4), 375-383.

[24] Parsons T.D., Courtney C. (2011). Neurocognitive and psychophysiological interfaces for adaptive virtual environments. In C. Röcker \& M. Ziefle (Eds.), Human Centered Design of E-Health Technologies (pp. 208 - 233). Hershey: IGI Global.

[25] Berka C., Levendowski D. J., Lumicao M. N.,Yau A., Davis G., Zivkovic V. T. (2007). EEG correlates of task engagement and mental workload in vigilance, learning, and memory tasks. Aviation, Space, and Environmental Medicine, 78, B231-B244.

[26] Brookings J. B., Wilson G. F., Swain, C. R. (1996). Psychophysiological responses to changes in workload during simulated air traffic control. Biological Psychology, 42, 361-377.

[27] Kobayashi N., Yoshino A., Takahashi Y., Nomura S. (2007). Autonomic arousal in cognitive conflict resolution. Autonomic Neuroscience: Basic and Clinical, 132, 70-75.

[28] Fairclough S. H., Venables L. (2006). Prediction of subjective states from psychophysiology: a multivariate approach. Biological Psychology, 71, 100-110.

[29] Pope A.T., Bogart E.H., Bartolome D.S. (1995). Biocybernetic system evaluates indices of operator engagement in automated task. Biological Psychology, 40, 187-195.

[30] Seery M.D., Weisbuch M., Blascovich J. (2009). Something to gain, something to lose: The cardiovascular consequences of outcome framing. International Journal of Psychophysiology, 73, 308-312.

[31] Bradley M.M., Lang P.J. (2000). Affective reactions to acoustic stimuli. Psychophysiology, 37, 204-215.

[32] Cuthbert B.N., Bradley M.M., Lang P.J. (1996). Probing picture perception: Activation and emotion. Psychophysiology, 33, 103-111.

[33] Cuthbert B.N., Schupp H.T., Bradley M.M., Birbaumer N., Lang P.J. (2000). Brain potentials in affective picture processing: covariation with autonomic arousal and affective report. Biological Psychology, $52,95-111$

[34] Hancock P.A., Szalma J.L. (2003). The future of neuroergonomics. Theoretical Issues in Ergonomics Science, 44, 238-249.

[35] http://graphics.cs.brown.edu/games/G3D/.

[36] Chance S.S., Gaunet F., Beall A.C., Loomis J.M. (1998). Locomotion mode affects the updating of objects encountered during travel: the contribution of vestibular and proprioceptive inputs to path integration. Presence, 7(2), 168-178.

[37] Steinicke F., Bruder G., Hinrichs K., Lappe M., Ries B., Interrante V. (2009). Transitional environments enhance distance perception in immersive virtual reality systems. In Proceedings of the 6th Symposium on Applied Perception in Graphics and Visualization, 1926.

[38] Campbell D.T., Fiske D.W. (1959). Convergent and discriminant validation by the multitrait-multimethod matrix. Psychological Bulletin, 56, 81-105.

[39] Miller L.S., Rohling M.L. (2001). A statistical interpretive method for neuropsychological test data. Neuropsychol. Review, 11, 141-167.

[40] Courtney C.G., Dawson M.E., Schell A.M., Iyer A., Parsons T.D. (2010). Better than the real thing: Eliciting fear with moving and static computer-generated stimuli. International Journal of Psyhophysiology, 78, 107-114.

[41] Macedonio M., Parsons T.D., DiGuseppe R., Weiderhold B., Rizzo A.A. (2007). Immersiveness and physiological arousal within panoramic video-based virtual reality. Cyberpsychology and Behavior, 10, 508-516.

[42] Phillips L., Interrante V., Kaeding M., Ries B., Anderson L. (2012). Correlations between physiological response, gait, personality, and presence in immersive virtual environments, Presence: Teleoperators and Virtual Environments, 21(2), Spring 2012, 119-141. 